ate evaluation system for knowledge and results of the drills should be developed.

Keywords: contents; course; disaster; drills; education; Japan; outcome

Prebosp Disast Med 2005;20(2):s87-s88

\section{Evaluation of the Usefulness of the Fiberscope for Confirmation of Intubation}

Y. Koido

Nippon Medical School, Japan

Introduction: The position of the tracheal tube in tracheal intubation is confirmed by the primary and secondary confirmation methods. However, incorrect intubation (esophageal intubation) can not be detected perfectly by these methods. Prehospital tracheal intubation often results in incorrect intubation.

Objectives: A fiberscope (FS) was developed for the confirmation of intubation, and its usefulness for confirming the position of the tracheal tube was evaluated.

Methods: The position of the tracheal tube was confirmed using a FS in 30 patients who underwent tracheal intubation in the emergency room.

Results: Since one of the 30 patients showed esophageal intubation, tracheal intubation was performed 31 times. The tube was confirmed to be in the trachea in 28 of the 31 cases, and in the esophagus in one case. The position of the tube could be confirmed in $29(93.5 \%)$ of the 31 cases. In two cases, the tube position could not be confirmed because of an inadequate visual field due to airway secretion, but reintubation after tracheal aspiration allowed confirmation of the tube in the trachea. The mean time required for confirmation was $7.1 \pm 3.1$ seconds.

Discussion: At present, the apparatus used by emergency medical technicians for the determination of esophageal intubation has a sensitivity of about $70 \%$, causing many false-negative cases. Though the protocol indicates confirmation after pharyngeal re-exposure when emergency medical technicians are not confident after primary and secondary confirmation methods, this procedure is difficult. In such a situation, the FS, associated with a high accurate confirmation rate, is useful.

Keywords: accuracy; emergency medical technician (EMT); fiberscope (FS); intubation; patients; prehospital

Prebosp Disast Med 2005;20(2):s88

Neglected Protection Against Weather Influences during Mass-Casualty Incidents: Lessons Learned in Field Exercises

\section{Mattila; A. Immonen; J. Holopainen}

Department of Physiology, University of Kuopio, Finland

This report includes the authors' personal experiences from several multiple casualty incident (MCI) exercises during different seasons in Finland. The conduct of drills is an established way to test the field preparedness of health services for real-life events, in order to minimize deviations from the accepted guidelines and avoid tactical and technical mistakes.

From a trauma care perspective, the goal is to provide "severely injured" subjects with simulated procedures approximating emergency care under real circumstances. This also includes avoidance of accidental hypothermia. Even for "smart casualties", it is difficult to simulate accurately the different effects of traumas on vital signs, with an exception of body temperature. Many subjects of drills actually are cold, pale, cyanotic, and shivering, when waiting for triage, decisions, care, and transportation. Routine measures for protection against cold, which include placing blankets and aluminized plastic foil on the casualty, seem insufficient because subjects are mostly immobile and lying on the cold and moist ground. Thermal imbalance is due mainly to heat conduction to the ground. When lessons are drawn from each drill during end-of-drill discussions, it would be important to include experiences of the "smart casualties". Unofficially, "terrible cold" often is reported as the most unpleasant experience, despite wearing exceptionally warm clothing.

The last two field drills were designed to correct the neglected protection from the weather by supplying a specially designed protective covering (TelesproTM) for the emergency group to be utilized on the subjects during the exercise. Before the drill, the group members were informed as to the purpose, characteristics, and use of TelesproTM protective covering. Despite this fresh briefing, the utilization of the protection remained only partial. Therefore, much more could be done in terms of protection of casualties against weather influences, if the existence of accidental hypothermia and its negative effects becomes generally acknowledged. It also is possible to simulate cold ambience and demonstrate protective isolation needs. An ongoing systematic education and evaluation for incorporation of improved weather protection is recommended.

Keywords: analysis; field exercise; hypothermia; protective clothing; smart casualties; trauma; weather Prebosp Disast Med 2005;20(2):s88

\section{Systematic Review of Prehospital Endotracheal Intubation in Trauma Patients}

A. Sen

Manchester Royal Infirmary, United Kingdom

Background: Ventilation and prevention of aspiration are of vital importance for trauma patients. Airway is the " $\mathrm{A}$ " of "ABC" in advanced trauma life support (ATLS). Endotracheal intubation (ETI) remains the gold standard for trauma airway management. The role of ETI in out-ofhospital ATLS, however, remains controversial. Recently, an increase in mortality has been documented in association with paramedic rapid sequence intubation (RSI) of severely head-injured patients. Some experts say that early field intubation was associated with a decreased risk of fatal outcome compared with emergency department intubation, while others claim ETI confers no survival advantage over bag-and-mask ventilation (BMV) and slightly increases prehospital time. Adequate oxygenation is important to the critically injured patient to avoid secondary damage. This analysis explores benefits and harms of prehospital ETI in victims of traumatic events by a systematic review of primary studies. 